\title{
Errors in short-term memory for number values*
}

\author{
DENNIS H. HOLDING \\ University of Louisville, Louisville, Ky. 40208
}

The effects of number values are explored in a pairing task which requires the maintenance of a rurning span. Performance improved over three trials, the final span reaching 7.6 items. Errors were classified as mistakes, failures, or repeats. As number values increased, the corresponding failure errors rose and fell, simulating a "serial position" curve, although repeat errors increased linearly, in conformity with earlier findings. The apparent divergence is ascribed to a differential involvement of recall and recognition processes. Both cases demonstrate the importance of number values in determining the efficiency of short-term storage.

Highly overlearned associations, such as those of the integral number series, seem to impinge upon memory processes even when interpretation of the stimuli is not required. In one instance, the serial properties of number were shown to influence the accuracy of performance in a recognition test of memory conducted by Thomas (1968). Although lists of two-digit numbers were presented in random order, the distribution of memory errors rose between the numbers 0 and 63 , falling to 99 in a typically bowed serial position curve. The presentation of the numbers was auditory, although visual recognition was required, while the retention in terval was relatively long.

Even with verbal response to an auditory input, and a minimal retention time, there is evidence to indicate that single digits pose differing problems for short-term memory. In a digit-span task with immediate recall, Holding (1970) found error probabilities increasing with the numerical size of the seven digits composing the lists; thus, lists with a mean of 5.5 appeared to be several times as difficult as lists with a mean of 3.5. These results emerged incidentally and have not been replicated, but suggest that difficulties in processing are brought about by the more complex arithmetical properties of the higher digits.

The effects of digit properties are further investigated in the present experiment, which makes use of the number-matching game known as "Concentration" or "Pelmanism." The game demands a running memory span for visually presented card numbers, over a relatively long but unbroken retention period. The use of a visual input increases the probability of

\footnotetext{
* The author is grateful to Dr. William A. Cook and to Charles F. Schanie for
} assistance in statistical analysis. demands upon longer term storage, with the possibility of éliciting material additional to that required for translation into auditory representation. Also, in a running span, items must be continually discarded from temporary storage. Since the basis for selection may vary depending upon the nature of the associative material retrieved from store, the method offers a potential assessment of any bias due to number properties in determining which items are retained.

\section{SUBJECTS}

There were 20 Ss of whom 10 were freshman students serving under course requirements and 10 were volunteer graduate students.

TASK AND PROCEDURE

The task was a modified version of the game. The Kings, Queens, and Jacks were removed from a deck of playing cards (Bee; Back No.67), and the remaining 40 cards were spread face down on a table in a randomly positioned array within the reach of a seated $\mathrm{S}$.

Performance was paced by soft 0.25 -sec "bleeps" at $1,000 \mathrm{~Hz}$, occurring at 5 -sec intervals. Upon one such signal, the $\mathbf{S}$ turned a card face upwards; on the next, he reversed it, replacing it in its original position. Proceeding in this way sooner or later resulted in the second member of a number pair being displayed. At S's discretion, this card could be left upturned while an attempt was made on the next signal to retrieve the first member of the pair. If successful, $S$ removed the two matching cards from the array on the next signal, stacking them at the side of the table; if unsuccessful, both cards had to be replaced face downwards and, on the following signal, another card was selected. A trial continued in this way until all 20 possible pairs had been matched and set aside.

All Ss performed three complete trials with repeatedly reshuffled decks, following an initial instruction period. This comprised: explanation of the task objectives and procedure; demonstration by $\mathrm{E}$ of the procedure and the pacing technique; paced practice by $S$ with a restricted set of 10 cards, different for each S. All Ss were able to match the pacing rate.

Each move made by $S$ during the test trials was recorded, in a notation which identified individual cards exposed, and which showed whether they were subsequently reversed, unsuccessfully left upturned, or paired.

\section{RESULTS}

Overall Performance

Although the task appears more difficult than straightforward digit repetition, the mean span during practiced performance on the final trial was of 7.6 items; "span" here refers to the mean number of intervening cards across which a pair was successfully achieved.

Performance improved from trial to trial in terms of combined errors. These were computed by counting the number of card-exposure moves in excess of the minimum of 60 moves which are required to make all the pairings. The mean error scores decreased from 21.3 (S.E. \pm 3.1 ) on Trial 1 through $14.2( \pm 2.4)$ to 12.5 $( \pm 2.9)$ on Trial 3. However, the pattern of relationships between card number value and error type and the distribution of error types remained the same from trial to trial; hence, the data from all three trials has been used in the subsequent analyses. Number Effects

A S playing this experimental game may commit three types of overt error. These may be arbitrarily designated failures, mistakes, or repeats and are best illustrated by example. Assume the following sequence of play (C, clubs; D, diamonds; $H$, hearts; $S$, spades): $S$ lifts

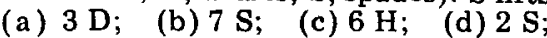
(e) $9 \mathrm{C}$; (f) $7 \mathrm{H}$; and proceeds (g) $4 \mathrm{D}$; (h) $6 \mathrm{C}$, leaving it faced; (i) $2 \mathrm{~S}$, and reverses both; (j) $9 \mathrm{C}$, no action appropriate; (k) $6 \mathrm{H}$, and pairs with (l) $6 \mathrm{C}$.

Move (f) represents a failure, since he could have paired the $7 \mathrm{H}$ with the $7 \mathrm{~S}$ exposed on Move (b). Leaving the $6 \mathrm{C}$ faced on Move (h), while unable to pair it, is a mistake which will add to the number of moves needed to pair the $6 \mathrm{~s}$. Since the card mistakenly exposed on Move (1) is a $2 \mathrm{~S}$ which has appeared previously, Move (i) will add to the number of moves required to pair the 2s and must be accounted as an error, taking the form of a repeat. An uncomplicated repeat occurs on Move $(\mathrm{j})$ when the $9 \mathrm{C}$ is exposed for the second time.

The three kinds of error appear to have differing kinds of dependency 

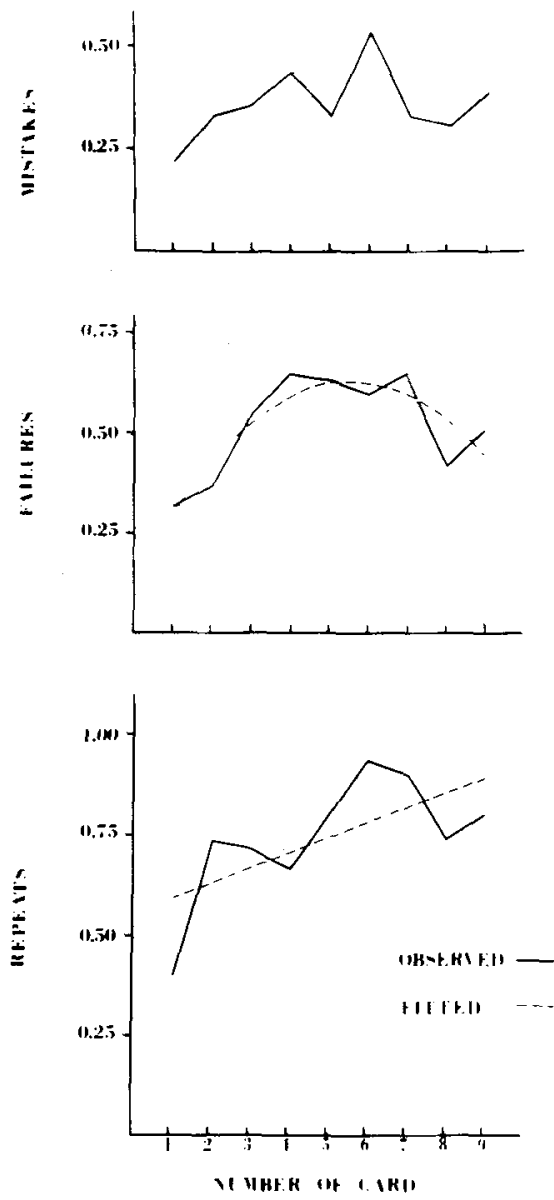

Fig. 1. Observed and fitted relationships between card number values and mean mistakes, failures, and repeats.

upon the number values of the playing cards. Curves relating the frequencies of mistakes, failures, and repeats to the number values are shown in Fig. 1. It was predicted that the results for the 10 s would be anomalous since, aside from possible end effects, they share some properties of $0 s$ and 1 s and are computationally easy; these numbers were intermediate on all measures and have been omitted from the analyses and figure.

In the case of mistakes, which constitute only $23 \%$ of the errors, there is no clear trend. Most cards wrongly chosen are cards which have been previously exposed; only $9 \%$ are new cards. Of the old cards wrongly chosen, most are serially adjacent to the correct card. Since most Ss follow consistent, although individually different, search patterns, serial effects are confounded with spatial propinquity. Both these factors will combine to mask the effects of number properties and of acoustic or visual confusions; constructing confusion matrices, showing what was confused with what, thus shows nothing of significance.

Failures to turn when an earlier card of the same number was available, forming $32 \%$ of all errors, show an extremely systematic effect. The relationship shown is a clear curvilinear trend of the "serial position" type, despite the fact that the cards were not presented to the $S$ in a numerical series. Analysis by linear models using orthogonal polynomials (Mendenhall, 1968) shows a significant quadratic component $(p<.05)$, with no significant linear trend. Differences between trials are also significant, but are of no immediate interest. The fitted curve appears in Fig. 1.

Repeats account for the largest proportion of errors, at $45 \%$. In contrast to the data on failures, the repeats show a less regular but basically linear trend. The Mendenhall analysis yields a significant linear component $(\mathrm{p}<.02)$, with a very small (-.088) and not significant quadratic term. The data on these errors appear, therefore, to confirm the Holding (1970) finding that increasing error results from increasing digit size.

\section{DISCUSSION}

In general, these results support the earlier conclusion that the digits are not processed as homogeneous symbols. The efficiency with which they are "borne in mind" appears to differ systematically as a function of their numerical values. However, it is also probable that more than one function is involved. Mistakes yield no consistent relationship with number, for the reasons already discussed, but failures show a "serial position" effect and repeats appear linearly related to digit size.

It seems probable that card location is better retained than number value, although the task involves serial retention of number and location as implicit paired associates, with either item potentially constituting the stimulus member. A $S$ may make a mistake in retrieving the earlier card of a pair because he confuses its number value with that of another or because he forgets where the correct card is located. He might fail to turn up a prior card because its location is uncertain or because he forgets that it exists. He might pick up the same card repeatedly because he forgets that a card in a given location is one which has been exposed, because he confuses the numbers of two known cards, or more probably because he forgets the number of a card known to have been exposed. These differing possibilities appear to lead to a divergence between failures and repeats.

The curvilinear relation for failures was obtained, like the "serial position" effect for numbers observed by Thomas (1968), when the numbers were not presented in serial order. A partial explanation would be available, were it the case that $S$ s restructured the items into serial order during rehearsal. Thus, rather than remembering that an ordered series of known locations contained the numbers $6,4,9,5$, and 2 , it is possible to attempt remembering the differing locations appropriate to the ordered numbers $2,4,5,6$, and 9 . However, interrogating $S s$ at the end of the experiment, and listening to occasional overt rehearsal, shows that Ss overwhelmingly prefer the first method.

What appears as a serial position curve must therefore be attributed to anchor effects at the ends of a subjective number scale. Recognition memory for the number values as stimulus items, and hence for the associated locations, appears less reliable in the case of the less distinctive middle numbers; extreme digits seem more prominent, and have a lower probability of discard from store. Failure to recognize a number as having previously occurred rather than failing to recall the earlier location may therefore be the primary cause of failure errors.

Distinguishing between the stimulus properties and the associative properties of digits in number-location pairs seems to afford a possible explanation for the difference between repeats and failure errors. It appears that, if the digits at the high extreme acquire distinctiveness in the context of the experiment, they remain low in associative potential. Battig \& Spera (1962) obtained norms, based on ratings of the number of associations and the ease of their formation, for the numbers 0-100. Extracting the association values for the single digits shows an approximate downward trend against number value which is in good correspondence with the present data for repeats. Computing rank correlations between the association value and the three error measures of this experiment yields appreciable and significant agreement with the data on repeats (rho $=-.61 ; \mathrm{p}<.05$ ).

The linear trend of the data for repeats is therefore most readily attributed to increasing associative difficulty. This hypothesis is broadly consistent with the explanation advanced for the digit span findings (Holding, 1970), where the associative problem appeared to lie in organizing the digits in combination. In the present case, the difficulty with repeat errors will occur primarily in associating number with location. Thus, for any known location, the corresponding numbers appear increasingly difficult to retrieve as 
their values rise. Faced with a familiar card, the $S$ is therefore less likely to recall its number when that is high, and more likely to commit repeat errors.

The failure errors are therefore seen as primarily due to difficulties of recognition, while the repeat errors are attributed to problems of associative recall. Although these hypotheses clearly call for further experimentation, the central finding depends upon trends common to both sets of error data. Thus, despite the apparent divergence in the forms taken by the relationship between errors and number values, the data provide further grounds for concluding that individual digits differ in the difficulty with which they are processed and remembered.

\section{REFERENCES}

BATTIG, W. F., \& SPERA, A. J. Rated association-values of numbers from 0-100. Journal of Verbal Learning \& Verbal Behavior, 1962, 1, 200-202.

HOLDING, D. H. Digit size in short-term memory. Quarterly Journal of Experimental Psychology, 1970, 22, 256-261.

MENDENHALL, W. Introduction to linear models and the design and analysis of experiments. Belmont. Calif: Wadsworth, 1968.

THOMAS, H. B. G. A serial position effect in number recognition. Joumal of Experimental Psychology, 1968, 78, 8-14.

\section{CURRENT LITERATURE ON HUMAN MEMORY}

CAREY, P. W. (Lexington School for the Deaf and Graduate Center, City University of New York, New York, N.Y. 10036). Verbal retention after shadowing and after listening. Perception \& Psychophysics, 1971, 9, 79-83.

DEICH, R. F. (California Department of Mental Hygiene, Pacific State Hospital, Pomona, Calif. 91768). Children's perception of differently oriented shapes: Word recognition. Perceptual \& Motor Skills, 1971, $32,695-700$.

DORNBUSH, R. L. (New York Medical College, New York, N.Y. 10029). Delay of auditory input in "simultaneous" auditory and visual short-term memory. Perception \& Psychophysics, 1971, 9, 97-98.

FITZGIBBONS, D. J. (Institute of Living, Hartford, Conn. 06102), \& GOLDBERGER, L. Task and social orientation: A study of field dependence "arousal" and memory for incidental material. Perceptual \& Motor Skills, 1971, 32, 167-174.

HALCOMB, C. G. (Texas Tech University, Lubbock, Tex. 79406), MCFARLAND, B. P., \& DENNY, N. R. Monitoring performance with a time-shared memory task. Perceptual \& Motor Skills, 1971, 32, 347-351.

HALWES, T., \& JENKINS, J. J. (University of Minnesota, Minneapolis, Minn. 55455). Problem of serial order in behavior is not resolved by content-sensitive associative memory models. Psychological Review, 1971, 2, 122-129.

HURST, P. M. (Institute for Research, State College, Pa. 16801), \& MCKENDRY, J. M. Effects of redundancy on performance under overload stress. Perceptual \& Motor Skills, 1971, 32, 907-915.

KROES, W. H. (Ohio State University, Columbus, Ohio 43210), \& LIBBY, W. L. Conceptual encoding categories in free recall. Perceptual \& Motor Skills, 1971, 32, 167-269.

KRUEGER, L. E. (City College of the City University of New York, New York, N.Y. 10031). Effect of direction of sequential presentation and redundancy on short-term recognition memory. Perception \& Psychophysics, 1971, 9, 121-124.

LOCKE, J. L. (Children's Research Center, University of nlinois,
Champaign, Ill. 61820). Acoustic imagery in children's phonetically mediated recall. Perceptual \& Motor Skills, 1971, 32, 1000-1002.

SMITH, N. C. (Ohio State University, Columbus, Ohio 43210). Long-term retention of a pursuitmeter skill. Perceptual \& Motor Skills, 1971, $32,773-774$.

WHITE, M. J. (Victoria University of Wellington, Wellington, New Zealand). A parallel between single-letter and multiple-letter recall of tachistoscopic letter displays. Perceptual \& Motor Skills, $1971,32,402$.

WILLIAMS, W. N. (University of Florida, Gainesville, Fla. 32601), \& LAPONTE, L. L. Intra-oral recognition of geometric forms by normal subjects. Perceptual \& Motor Skills, 1971, 32, 419-426.

ZAKIA, R. D. (Rochester Institute of Technology, Rochester, N.Y. 14623), \& HABER, R. N. Sequential letter and word recognition in deaf and hearing subjects. Perception \& Psychophysics, 1971, 9, 110-114. 